\title{
DER SÊLE CRANZ.
}

Swer sich zu gote wil kêren, einen list wil ich in lêren, wî er sîn dinc sulle ane vân, daz her gotis hulde muge hân:

5 wî er daz sulle beginne, daz her daz himelriche gewinne.

Der êrste unt der beste rât, den man an der schrift hât, der ist geheizen alsô:

10 vêra cordis contriciô. daz sal man alsô verstân: man sal ganzce rûwe bân unde bitterlíche smerzcen tragen in deme herzcen

15 umb die sunde unt umb die missetât, die der lîp begrangen hât.

Ueberschriften: Ditz bvehel heizet der tvgētkrātz Daz mach vns an der sele glantz (rot) $B$, Der krantz der gotlicher liefrden $C$. 1-4 fehlen $C$. 1. 2. Swer $\mathrm{zv}$ got sich keren wil Einen list ich in leren wil $B$. 2. Eine $A$. 3. dinc sin mit den gervöhnlichen zeichen der umslellung $A$. an $B$. 4. er daz himelrich $B$. 5. 6 fehlen $B$. Hier beginnet der kranz der minnen wie man dat hemelrich sal gewinnen $C$. 7. Kein absatz $A B C$. und ouch der leste $C$. Den ersten un den besten r. $A$. S. inder $B$. Als men in der heilgen s. h. $C$. 9. Der fehlt $C$. 11. sol $B$. 12. ganzen ruwen $C$. Daz man gantze riwe so han $B$. 13. Vnd bitter $C$. bitterlichen $B$. 14. Sal men drag. $C$. $\operatorname{dem} B C$. 15. Vmbe $B$. dic swaren sunden $C$. un $A$, vnde $B$, und $C$. umb die fehlt $B C$. 16. lip] minsche $C$. 
swenne sô daz ist geschên, sô sal man der sunde vorjên:

mit weinen unt mit grôzer clage

20 sal man sie deme prîstere sage:

herzce unt ougen sullen weinen.

sus sal sich der mensche reinen.

dar nâch sal die bûze gân.

die sal man vrôlîchen entfân

25 unt sal sie tragen an die zcit,

daz der mensche tôt lît.

wirt her an der bâze vunden,

wol in der lieben stunden,

daz in sîn mâter ie getrûe;

30 got gibt ime aller wunne gnûc,

der sîn herzce kiesen wil

beide âne ende unt âne zcil.

swer zû der vroude wirt erkorn:

wol ime, daz er ie wart geborn.

35 Wilch dise bûze sulle sin, daz wiset uns diz bûchelîn.

die ougen sullen vlîzen

unde heize trêne gîzen

unt gote inneclichen clagen,

17. Wenne $A$, Wanne $C$. sô fehlt $C$. geschehen $B$, also geschein $C$. 18. So svlle wir $B$. der sunden v'iehen $B$, die sunden verghein $C$. 19. schreien $C$. groizen $C$, fehlt $A$. ching $B C$. 20. Sol $B$. si $B$, fellt $C$ '. $\operatorname{dem} B$, den $C$ '. prister $B$, preister $C$. sagen $B C$. 22. Also $B$, Alsus $C$. sich fehll $C$. minsch $C$. von (syn) sunde reynē $A C$. 2.3. ergan $B$. D. n. s. men zo der bicht gain $C$. 24. vrolich $B$. Und vur dic sunden penitentie entfain $C$. 25. sal sie fehlt $C$. biz an $B C$. 26. Biz daz $A$. Als he den duit leit $C$. 27. Uñ wirt $A$. in penitencie $C$. erfvnden $B$, gefunden $C$. 28. So wol $A$. im zv den st. $B$, im dan der vroelicher st. $C$. 29. ym $C$. 30. im $B C$. aller] dan $C$. vrevden $B C$. $31-34$ fehlen $C$. 32. Ane zal vnd $B$. 33. Wer $A B$. den vrevden $B$. 34. im B. 35. Kein absatz BC. Welch (sonst stets wilch) $A$. Wie aver die penitentie sal $\sin C$, Wie aber die sei gestalt $B$. 36 . boechelgin $C$. Daz svlt ir prvfen manicvalt $B$. 37. sleissen $C . \quad 35$. Und daz herze $B$, Und van $C$. tranen $C$. giezen $B C$. 39 . got $B$. mynneclichen $A$, innenklichen $B$, innichlichen $C$. 
40 daz die sêle in sunden lît erslagen.

weinen ist sô ein gût dinc, daz Jhêsum, der meide kint, niemant sô wol erbiten mac, sô der dâ weinet nacht unt tac.

45 an deme buche geschreben ist von unseme herren Jhêsû Crist, daz sîn reiner kûscher munt gelachte nie zu keiner stunt. wir vinden ouch geschriben, daz

50 sîne ougen worden dicke naz unt sîn vil schônen wangen mit heizen trênen bevangen. ôwî unde owê unde owê, hate unt iemer mê!

55 wilch rât wirt unser danne, daz wir sô gerne zanne unde alsô gerne lachen unt deme tâvele vroude machen? der vil sûze geweinete trân

60 der gêt vor Jhêsum Cristum stân unde versunet den sundêr vor deme zornigen richtêr. daz tât alliz des sunders trân: sô wol ime, der in mac gehân.

40. die] $\sin B$. in den sund. $A B$. mit sunden "si gesl. $C$. 41. Want w. $C$, sô fehlt $B C$. svze dinch $B$, soez $\operatorname{dink} C$. 42. iesum christvm $B$. Dat Jesus Christus Marien k. $C$. 43. Nieman $B$, Ghein man $C$. gebidden $C$. 44. da fehlt $B$. schriet $C$. unde $A$, vñ $B$, und $C$. 45. In den boichen $C$. In der schrift man list $B$. 46. unserm $B$, unsen $C$. ihesvm $B$. 47 . reine ware mont $C$. 48. Nie gelachte $B$, Nie enlachten $C$. cheiner $A$, einiger $C$. 49. 50 fehlen $C$. 50. Daz sin o. wurden naz $B$. 51. sine $A$. vil schonen] minnenclichen $B$, sueze $C$. 52. heizen fehlt $C$. trehen $B$, tranen $C$. vmbe vangen $B$, al umbhangen $C$. 53-108 fehlen $C$. 53 . Owe vnd o. $B$. 54. Wafen geschreit sei immer me $B$. 55. Welch r. sol u. werden dannē $B$. 56. zannen $A B$. 57. Vnd - gern $B$. 58. Uñ $A$, Vnde $B$. dem tevfel $B$. 59. vil] klare $B$. geweinte (ge ist von alter hand übergeschrieben) $B$. 61 fehlt $B .62 . \mathrm{Zv}$ dem zornigem gerichte $\nabla \tilde{n}$ fvrchtet sich vor nihte $B$. 63. Ditz tvn allez d. s. trehen $B$. 64. Sô fehlt $B$. ime $A$. in] dich $B$. gehân] sehen $B$. 
65 unde ôwê herre Jhêsû Crist, daz mir der trân sô selsen ist:

daz sî dir herre got geclair unde Marîen, der kûschen mait. vil sundigen ougen mîn,

70 wî lange wolt ir trocken sin? ir beitit al $\mathrm{zu}$ lange. begîzet mir die wange. von weinen sult ir wesen rôt; die sêle lît in sunden tôt.

75 swer sus kan gebâren, der sêle sô wol mite gevaren, daz ist der bûzen eine, die dî sêle machit reine.

Die andere bâze ist sô gestalt,

$80 \mathrm{daz}$ dâ tegelich gedenken salt an die martẹr unt an die nôt unde an den jêmerlichen tôt unde an die grôzen arbeit, dî her durch dînen willen leit.

85 dine sunde wolde her tragen, dô her an daz crûzce wart geslagen. dar an stûnt her nackit unde blôz:

daz blût ron sîner sîten vlôz, von vâzen unt von henden

90 af die erde allen enden; sin lîp was allenthalben wunt, ouch was sin rôsen rôter munt

65. Eya svze $B$. herre fehlt $B$. 66. daz weinen $B$. sô fehlt $B$. seltzen $B$. 67. 68. D. sei d. svzes kint gekleit vnd dir moter reine meit Marien der kvnegin aller sünder ein trösterin $B$. 70. welt $B$. trveken $B$. 71. alze $B$. langen $A$. 72. wangen $A$. 73. werden $B$. 74. die leit $B$. in den 8. $A B$. 75. 76 fehlen $B$. 77. bvze $B$. 79. ander $B$. 80. teglich $B$. 82. bitterlichen $B$. 84. her got $B$. 85. wolt er $B$. 86. $\mathrm{Da} A B$. er wart an d. c. g. $B$. 87. hiench er $B$. 88. sinen $B$. 90. erden $B$. 91. wart allen enden $A$. 92. wart $B$. rosen varber $B$. 
beide varwelôs unt blêch:

daz houbit ime af die erden wêch.

95 ezic wart ime geschenket, mit gallen wart he getrenket:

an allen leden glich

wart her gemartert durch dich. daz têt her alliz umbe daz,

100 daz dû dîne sunde deste baz woldest kêren an sîne êre. Marîa, kuneginne hêre, wie was deme herzcen dín, dô dû dîn lîbez kindelîn,

105 unsen herren Jhêsum Cristum, dînen eingebornen sun, an deme crûcze sêhe hangen mit biûte gar bevangen? vil reine kuneginne,

110 wilch wâren dîne sinne in deme grôzen herzceleide unt in des jâmers ougen weide? ich spreche daz mit wârheit, daz nie mûter geleit

115 alsô grôze smerzcen

an lîbe noch an herzcen, alsô dî tête an den stunden umb dînes lîben kindes wunden. ich vil sundiger mensche, waz sal ich?

93. heide fellt $B$. unde $A B$. bleich : weich $B$. 94. Sin houbt im uf sin achsel w. $B$. 96. er $B$. 97. liden gelich $B$. 99. allez dveh daz $B$. 100. dine sunde] in $B$. dester $B$. 101. Soldes halden vnde keren an sin lop vnd an $\sin$ eren $B$. 102. 3. M. svze kvnegin Sag mir wie w. dem h. d. $B$. 104. Da $A$. 104. 105. Da du ihesum xpm. 106. einen geb. $A$. Den lieben dinen einen sun $B$. 107. $\operatorname{dem} B$. sehest $A$. 109-112 fehlen $B$. 109. O edel koeninginne $C$. 110. Wat deden $C$. 111. dem gr. bitteren Jyde $C$. 112. der iemerlichen (jannerlicher $C$ ) $A C$. 113. sprechen $C$. mit der $C$, bi der $B$. 114. nie kein $B$. dar enleit $C$. 115. 116 sind in $C$ umgestellt. 115. Al sulchen $C$. grozen $B$. smerzce: hercze $A$. am - am $C$. 117. Alsoe $C$, Als $B$. dedest $C$. an] $\mathrm{zv} B$. 118. Umbe $A B$. 119. fehlt $B$. vil fehlt $C$. sundige $C$. mensche fehlt $C$. 
120 war sal ich vorbergen mich

an deme tage, sô Jhêsus Crist

an daz urteil zu kunftic ist

unde her urteil wil geben

uber mînen lîp unt mîn leben?

125 sô lêzt her mich sîn wunden sên.

eiâ, waz sal ich denne jên?

waz mac ich sprechen zû der zît,

sô iz vor mir geschriben lit,

daz ich sunder habe getân?

130 vor deme tage mûz ich angest hân.

swer dise wort unt dise dine,

die hie nû geschriben sint,

dicke in dem munde treit

und in sîme herzen uberleit,

135 daz ist der sêlen grôz heil

unt leschet sunden ein teil.

120. Wa sol $B$. verbergen $B C . \quad 121-122$. Als got an daz gerihte sich wil mit sinen heiligen kvmen als ich han ander schrift v'nvmē $B$. 121-124. In dem dage Jesus Christus unse here dan mit groizer maiestait und ere wirt komen zo ordelen over min lif und over alle man und wif nnd heischet rede van unser dait so wie sich der ergangen hait ein iglich vur dat sin alein he si daegroiz of clein he si arm of rich dat ordel gait dair gelich $C$. 122. kinftic $A$. 123. Vnd $u$. wirt gegeben $B . \quad 124 . \min B$. vñ vber $\min B .125 .126$ fehlen $B$. 125. Al dae $C$. laizt $C$, lezet $A$. uns $C$. sine $A$. 126. waz $A$. 0 we wes sullen wir dan beghein $C$. 127. macl sol $B$. ander zit $B .128$. Wen $B$. iz alliz $A$. 127. 2s. Alsoe vur uns stait geschreven die sunde die wir begangen haint mit hant und monde $C$ '. 129. D. i. ze svnden ie getet $B$, Und wie sie sint gedain $C$. 130. Vur den dage sullen wir sorch hain $C$, Mich enhelfe denne din gebet Sve kvneginn S', sol ich vbel gedingene So bin ich v'lorn immer mer Genade svze maget her $B$. 131-136 fehlen $A$. 131. 2. Soe wer dese dink und dese wort die ir hic vur hain gehoirt $C$. 131. dinch vint $B$. 133. Ducke $C$. dem] sinem $B$. 134. wail overlecht $C$. Vnde si in sin herze leit $B$. 135. !)az ist dir ein michel heil $B$. 136. Vnde lest $B$. cier sund. $C$. ein wichel t. $B$.

NOch sint ovch ander gvte werk, Da mit man zv himel vert.

Die sele wol ovch singen mak

Swenne so kvmet der letzte tack
5 Swen si von den libe scheidet vnd des letsten vrteils beitet. Daz ist zvht vnde kevscheit vude des libes reinikeit: 
Swilch mensche sich wil lâze

af die himelischen strâze, der vindet bî deme wege stân

140 edele blûmen wol getân, die ime den wec gebreiten unde in wol geleiten.

ir smac der ist sô gât, daz her Jhêsû Cristô samfte tât.

145 swer einen crancz bricht von den blûmen unde vlicht unt in vor Jhêsum bringet, eiâ, wie wol deme gelinget. die blûmen, die man dâ siht,

150 die wahsen in dem himel niht, îdoch sint sî dâ harte wert, want man ir dâ sêre gert. swer uf den wec zu himelrich kumen wil, der vlîze sich,

Daz ist der reine magetvm, 10 an hohvart vnd ane rvm.

Swen wo die zwei volgen nach, Daz ist der sele ein michel schach. Beten, vasten, wachen,

Daz tvt die heiligen engel lache 15 vn twinget si dar $z \nabla$,

Daz si spat $\nabla \mathbf{n}$ fru

vmbe den menschen mvzen varn,
Daz si liep vnde sele bewarn. Swer gerne horet gotes wort

$20 \mathrm{Da}$ von wirt er dvrch bort, Daz er die kevsheit enphet, Die vor gotes antlvtze get. Daz ist daz vrone himelrich Do (l. daz) geit got einem ietslich, 25 Der gerne horet von got sagen vndiz in sinem herzen wil trage. $B$.

137. Kein absatz $C$. Wilch $A C$, WElch $B$. minsch sich nu $C$. lazen $B C$. 138. Hin of $B$. himelische $A$, rechten homelsohen $C$, himel $B$. strazen $B C$. 139. dem $B C$. 140. Zwelf $A$, Menich edel $C$. bloemgin $C$. 141. im $B$. eme einen sachten wech $C$. bespreiten $B C$. 142. Vnd senften wek (einen soezen gank $C$ ) bereiten $B C$. 143. 4. Ir smach und roich is wunnenclich dat gelust gode van hemelrich $C$. 145-148 fehlen $C$. 145 . kranz da von dringet $B$. 146 fehlt $B$. 147. in] den $B$. ih̄̄ $\times \bar{p} \overline{\mathbf{m}} B$. 148. Der sol im willekomen sin vnde der liben mvter sin Marien der himel kvnegin vnd allen sinen engelin $\nabla \bar{n}$ heiligi $B$. 149-152 fehlen $A$. 149. da entsprigen siht $B$, heir suicht $C$. 150 . Si enwassen im hemelrich nicht $C$. 151. do $B$. Dair sint si wert $C$. 152. Wen $B$. si ser begert $C$. 153. 4. Dar vmbe so vleize sich Ein ietslich of den weck $\mathrm{z}$ himelrich $B$, Soe vlize sich dairzo ein iglich der dair begert den wech zom hemelrich $C$. 153. Wer $A$. 
155 daz her dî blâmen breche unt sich dâ mite besteche unt mache dâ von einen krancz, den her trage an der engel tancz, sô sint sie ime alle dînsthaft

160 durch der edelen blumen craft unde durch ire wirdekeit: wol ime, der in dâ treit.

Disse blamen wil ich â nenne, daz ir sie moget erkenne.

165 ein Kûscher Lî̀ mit dêmûtikeit, daz ist ein blûme sô gemeit, die harte werdis lobis ist vor unsen herren Jhêsû Crist unt vor der sazen kunegin

170 Marten, der mûter sîn daz wizzet endelíche $\mathrm{zu}$ vorderst in himelriche.

Noch stêt ein edele blume dâ, die heizet obedienciâ.

175 Gehôrsam heizt daz blûmelín,

155. dese soeze blomen $C$. Da er d. bl. br. vñ allez leit im da von ze breche $B$. 156. sich selves dair mit $C$. bestecke vñ sine sele er wecke $B$. 157. dair $C$. 158. engele $A$, megede $C$. 159. im aber alle (alle ist übergeschrieben) $B, \mathrm{im}$ al $C$. 160. Al durch die $C$. 161. Vñ $B$, Und $C$. ire] des kranzes $B C$. 162. Wol in $B$, Wail dem $C$. d. desen kranz dreit $C$. da uffe $A$. 163. Dje $B$. Nu wil ich uch dese bloemen $C$. nennen $B C$. 164. Up dat $C$. mvget $B$, recht leren $C$. erkennen $B$, kennen $C$. 165 . Ein kuscher munt $A$, Eine heizet lop $B$, Kuische liefde $C$. mit] und $C$. demuticheit $A$, Diemvtikeit $B$, oitmoedicheit $C$. 166. sô gemeit] daz si evch geseit $B$. Dese blomen sint angeseit $C .167-172$ fehlen $C$. 167. here werdis $A$, harte grozez $B$. 168. ihw $A$. In der kamer ihā k. $B$. 169. vor fehlt $B$. 170. Sente marien $B .171 .2$. Die blvme ist so schone In dem himelriche vrone $B$. 173. Kein absatz $A C$. Noch soe steit $C$. edele fehlt BC. aldae $C$. 174. Und heyscht $C$. 175. Gehorsam heizet $B$, Gehorsamekeit heizit $A$, Dat is gehoirsamheit heyscht $C$. bloemgin $C$. 
iz mûz ouch an deme krancze sin.

sîn ruch der ist sô sûzlích, iz gelustet got von himelrîch.

Zwû blûmen ir noch merken sult:

180 Einvaldikeit unde Gedolt.

sie sint mit den krenksten niet;

got sie selber gerne siet

vor sîme antlitze stân.

man mûz sie an deme kranze hân.

185 Ein edel blûme noch dâ stêt, die heizet BarmuERziKêt. ir gespil stêt dar bie:

ich wêne ez Miltiklit sie.

lâzet sie û nicht vorsmâu,

190 got wil sie mit den besten hân.

Sô man beginnet vorbaz gân zwâ blûmen vindet man dâ stân:

Kastigen unde Mâzn:

man sal sie nicht dâ lâze.

176. Darz $B$, Si $C$. ouch fehlt $B$. dem $B C$. kranz $C$. 177. 78. Si gift vur gode lichten gelanz und ziret wail der megede kranz $C$. 177 . Ir ruch $A$, Sin smack $B$. 178. Daz sin gel. $B$. 179. Kein absalz $A C$. Noch zwei bloimgin ir mirken 8. $C$. 180. Dat is einveldicheit $C$. un $A B$, und goit $C$. Darauf fulgen noch: Daz sint zwu edele blvmen $\mathrm{Si}$ smecken vns an dem grmen $B$. 181. ensint $C$. bi den $C$, beide die $B$. krenkisten $B$, krenken $C$. 182. Got selbe si vil gerne siht $B$, Als dat got si gerue van herzen sicht $C$. 153. sinem $B$, sinen $C$. antlvtze $B$, angesichte $C$. 184. an] ouch zo $C$. dem $B C$. klanz $C .185$. Kein absatz $A C$. Ein vrische bloeme noch dair steit $C$, F.In ander bl. stet do noch gemeit $B$. 1\$6. Und heyseht die $C$. barmeherzekeit $A$, barmherzikeit $B C$. 187. dâ $B$, nae dair $C$. bi $A B C$. 188. meine $C$. daz ez $B$, dat it die $C$. demuticheit $A$. si $B C$. 189-192 fehlen $C$. 189. Nu lazet $B$. veh $A$, evch $B$. v'smāhen (hen ist durchstrichen) $B$. 190. den werdesten $B$. 191. Kein absatz $A B$. gên : stên $B$. 193 . Absatz $B$. Kastigrnge $B$, Castigeren $C$. v̄̄ $\mathrm{B}$, zo $C$. mazen $B$, maizen $C$. 194. M. sol si doch niht lazen $B$, Sal men viet hinder laizen $C^{\prime}$. 
195 sie sint ouch an deme kranze gât,

ob in der mensche rechte tût.

Noch ist der krancz nicht volbracht. sûze Gebet unt reine Andacht, daz sint zwô edele blâmen,

200 die da wol smecken an deme gûmen; sô man sî leget in den munt, die sêle wirt dâ von gesunt.

Eine schône blûme stêt noch dort, die heizt Hôr GERNE GOTES worT.

205 sie gibt vor gote lichten glanz unt zîret wol der engel tancz. wir suln sî an der strâzen zu himele nicht lâzen.

Noch sint zwâ blûmen wol gestalt,

210 die bie den besten sint gezalt. von einer lese wir alsô, die heizet Mansuetûlô, die andere Taciturnitas, die gote in deme munde was.

215 die eine heizet Senftikeir, die ander heizet Stillekeit.

195. Sie is $C$. ouch fehlt $B$. zv dem $B$, kranz $C . \quad 196$. Als $C$.' in $B C$. minsch recht $C$. 197-21s folgen in $C$ nach 219228. 197. Kein absatz $A C$. Dese kranz en is noch neit $C$. vollen braht $B C$. 198. Innich $C^{\prime}$. rein $C^{\prime}$. 199. 200 fehlen $C^{\prime}$. 201\%. Si sm. vns an dem $B$. 201. 202 fellen $A$. Wer die draget in sinen mont der macht sin sele gesunt $C$. 203. Kein absatz $A C$. Noch soe steit ein blome durt $C$. EIn sch. bl. stet dort verre $B$. 204. Und heyscht $C$. heizet $A B$. hore $A$. gotes wort hore ich gerne $B$. 205. 216 sind in $B$ umgestellt. 205. Si gibet $B$. got $B$. schein $C$. 206. Di $B$. engele $A$. kran $\% B$. Ind moiz ouch an dem kraiz sin $C$. 207. 208 fehlen C. 207. sullen $A B$. 205. Niht hinderstellich 1. B. 209. Kein $a b$ salz $C$. Doch $\sin A$. sint fehlt $C$. Zwn edele bl. $B$. ?10. Die zv dem $B$, Mit den $C . \sin A$. si ge\%. $C$. 211. Von der einen $B$. Ich lese van der einen a. $C$. 212. Dat si heyseht $C$. 213. ander $B C$. 214. got ie mirnende $B$. Dic unse here lief havende was $C$. 215 . senfticheit $A$, sachtmoedicheit $C$. 216. stilleheit $A$, stillicheit $C$, stetikeit $B$. 
ir sult $\$ 1$ gerne brechen unt den kranz dar mite bestechen.

Noch hân ich eine blûme ersên,

220 der mâz ich alles gûtes jên.

die heizet Sunde weine

unde ist der besten eine;

wan got durch iren willen tût

alliz, daz sî dunket gût.

225 Noch stêt dâ, als ich wêne, ein blûme, die ist seltsêne:

T'rûwe heizt daz blûmelîn.

iz mûz ouch an deme kranzce sîn.

Nû tret wir vrôlîchen vor

230 in daz himelische tor.

dâ stên zwû blûmen hêre, der bedarf man harte sêre, daz man sie lege an den kranz, sô ist her vil nâch worden ganz.

235 Hoffnunae unde Geloube, ân dise $z w \hat{a}$ sint d' andern toube.

217. Man sol $B$, Men sal $C$. si ouch $C$. 218. Unde $A$, fehlt $B$, Und $C$. da mit bestecken $B$. 219. Kein absatz $A C$. have $C$. ein $C$. blume $A$. er sen $A$, ersein $C$, gesehen $B$. 220. D. wil i. vil nahen des besten iehen $B$, Gerne weinen van den besten eln $C$. 221.22 fehlen $C$. 221. sunde] gerne $B$. 222. Si ist $B$. 223. Want $C$, Wenne $B$. iren] der blomen $C$. 224. Allet wat der minsch begert und d. g. $C$. 225. Kein $a b$ satz $A C$. dâ fehlt $A$, do $B$. Noch so stait dair eine $C$. 226. Ein bloem as ich meine $C$. 227. Triwe $B$. heizit $A$, heizet $B$, so heyscht $C$. dat fin bloeimegin $C$. 228. Si moiz ouch mit an desem kranz sin $C$, Iz mach wol der besten eine sin $B$. 229. Kein absatz $A B$. Nu tretit her $A$, Nu gain wir $C$. vroelicher $C$. 230. $\mathrm{Zv}$ der himelischen $\operatorname{trr} B$, Al entegen des hemels duer $C$. 231. Dair $C$. here] ain gevere $C$. 232. bedarfft $C$. wail harde $C$. 233. si stecke (steche $C$ ) $B C$. 234. hie $B$, he $C$. nae $C$. 235. Daz ist hoff. $A$, Hoffen $C$. un $A$, vnde $B$, und $C$. gelouve sonder do $C$. 236. dise] die $B$. so $\operatorname{sint} A$. die $A B$. Dese synt die a. alle toube $B$. anderen due $C$. 
swer diser zweier blamen entpirt, gotis kint her nummer wirt.

Nâ wol hin an daz himelstor.

240 dâ stêt ein edele blùme vor, die selbe ist die leste unde ist die aller beste. swer zû der pforten sal in gân, der vint sî bî deme wege stân

245 halp ûzen unt halp inne: daz ist die wâre Minne. niekein blûme ist ir glîch : sie breitit sich in himelrîch, ouch gûten smac und edelen ruch

250 hât die blûme âne allen bruch. swie ez den andern ergê, dise ervalwet nummer mê. sie gebôt gote von himelrich, daz her durch uns lîz martern sich;

255 wan in die minne dar zâ twanc, daz he verkôs sînẹs vater lant unde durch uns leit den bittern tôt, als ime die wâre minne gebôt.

237. Soe wer $C$, Wer $A$. dirre $B$. 238. Godes vrunt $C$. nimmer $B$. enwirt $C$. 239. Kein absatz $A$. himelische tor $A$, ander tor $B$. $\mathrm{Na}$ gain wir vroelichen in die doir $C$. 240. Ind dair $C$. edele fehlt $B$, riche $C$. vur $C$. 241. Die blvme $B$, Dese bloeme $C$. die ist $A$. letst $B$, beste $A .242$. ist fehlt $B$. Und aller blomen b. $C$. best $B$, leste $A$. 243-246 folgen in $C$ nach 247-250. 243. Und wer $C$. sal] wil $C$. in sol gên $B$. 244. vindet $A B C$. inder tvr sten $B$, in der doeren staen $C$. 245. dair buizen $C$. unde $A$. halbe $B$. dair inne $C$. 246. w. godes minne $C$. 247. Nirchein $A$, Kein $B$, Ghein $C$. enis $C$. gelich $B$, gelijche $C$. 248. Want sie spreidet $C$. sich] sie $A$. in daz himelrich $B$, in dem hemelriiche $C$. 249. 50. Und gift uns up erden ouch soeze smach und guide rouch $C$, Si gibet vns vil svien smak vnd svzen rvch als si wol mack $B$. 251. 252 fehlen $C$. 251. Wie $A$. andern blvmen e. $B$. 252. versalwet $A$. nimmer $B$. 253. Die blvme $B$, Deser bloemen $C$. golvt (?) $A$, entboet $C$. got $B C$. 254. durch si liez $B$. Als doe he neder quam up ertrich $C$. 255. 256 fehlen $C$. 255. Avch in $B$. twanch $B$. 256. verlos $A$. 257. Und $B$. Ind leit vur u. d. bitteren d. $C$. 258. Soe $C$. im $B$. entboit C. 
swer an sînen schatehût

260 dise schônen blûmen tût

zû der andern blûmen schar,

sô ist der kranz bereitet gar.

swer alsô hie geringet,

daz her den kránz vor gote bringet,

265 der ist sâliclîch geborn

unde hât daz beste teil erkorn.

Eiâ, milde Karitas,

hilf uns in daz palas,

daz wir uns dâ gevrowen

270 unde got dar inne beschowen

unde die lîben mûter sîn,

die himelischen kunegîn,

die ist schône unt wunnenclich,

daz mac nû wol sprechen ich.

275 swer eine stunde solt dâ sîn, unde wêre die werlt rôt guldîn, die nême ich vor die vroude nicht noch vor daz wunneclîche licht. eiâ, milde Jhêsû Crist,

280 wî sâlic der geborn ist, der iemer bî dir wesen sol;

259-262 fehlen $A$. 259. schaten hrt $B$. Absatz $C$. Soe wer an desen edeln kranz unt hoet $C^{\prime}$. 260. Alle dese schone bl. doet $C^{\prime} .261$. Und tzo den $C$. 262. Dan is $C$. gemachet $C$. 203. alsvist $B$. hir $A$, fellt $B$. Soe wer nae desen bloemen ringet $C$. 264. Und sulchen k. $C$. vor gote bringet] vol bringet $B$. 265. Ind der $C$. selick $B$, wail selich $C$. 266. Und $C$, Er $B$. bezzer $B$. uizerkoren $C$. 267. mille] du vil soeze $C$. 268. daz schone (vroeliche $C$ ) p. $A$. 269. Da w. v. inne vrewen $B$. Dat w. u. dair inne moegen ervreuwen $C$. 270. gote $A$. Vnde got mozen beschovwen $B$, Ind gode van hemelrich moizen beschouwen $C$. 271-334 fehlen $C$. 271. 72. Vnde sine mvter sente Marien Die kvneginne vrien $B$. 273. so schone $B$. unde $A$, v̄̄ $B$. wūnenclich] liht $B$. 274. Man mag da von gesprechen niht $B$. 275. ein $B$. solde $A$, fehlt $B$. da mohte $\sin B$. 276 . Wer dise $B$. rôt fehlt $B$. 277. nem er fvi $B$. 278. fvr des himelriches $B$. 279. milde] svie $B$. 250. selick er $B$. 281. Der bi dir immer w. s. $B$. 
deme ist âne mâze wol.

swer dîn antlicze sohowen mûz,

deme ist aller sorgen bûz;

285 der mac vrô sîn ummer mê, want ime wirt dâ niemer wê. sîn herzce mûz in vrouden wesen, dû bist sîn lîp, dâ bist sîn genesen. swaz her gert von diner hant,

290 daz gibestû ime alzuhant; wan dû himels unde erde gewaldic bist unde allis, daz dâr inne ist. dâ schowet man die kunegîn sente Marien, die mûter sîn,

295 unde mangen engel schônen mit guldînen krônen unde ander heiligen gar vil, âne zcal unde âne zil. snê noch rife dâ gelît;

300 dâ ist summer zaller zît, dâ hât der winter keine gewalt. vil manic sûze brunne kalt in der wisen entspringet. die nachtegal dâ singet

305 unde ander cleine vogelin. sêt, dâ wolle wir gerne sîn. swen wir sulche mêre hôren lesen, sô wolle wir gerne zu himele wesen. doch sô kome wir sô gâhs nicht dar,

282. Dem $B$. ane zwivel $B$. 283. antlvtz $B$. 284. Dem $B$. 285. D. mag sin vro imm. m. $B$. 286. Wen $A$. Sine libe wert immer ane we $B$. 287. wirt inden vrevden sweben $B$. 288. genesen] leben $B$. 289. Swes $B$. 290. im $B$. 291. Wenne $B$. erden $A B$. gewaldic bis $A$, waldes $B$. 292. alliz $A$. Und allez richez haldes $B$. 293. Daz sch. an $A$. 294. die] der $A$. 295. Die macht die engele schone $A$. 296. Mit der $g$. crone $A$. 297. U. a. engel harte v. B. 299. 300 sind umgestellt $B .299$. da nimmer lit $B$. 300. zu aller $A$. D. i. orch summer allezit $B$. 301. Der winter hat da kein g. $B$. 302. lvter $B$. 304. da lvte s. $B$. 305 . and'e $A$. cleine] manich $B$. 306. En trvwen da wolte $B$. 307. Swenne $B$ 30. wolde $B$. himel $B$. 309. D. kvmt nieman so $B$. nahes $A$. nicht fehlt B.

Beiträge zur geschichte der dontschen uprache. V. 
310 daz wir beschowẹn der engel schar, wirn haben ez hie erarnet. got hât uns gewarnet. iz sî man oder .wîp, iz sal kastigen sînen lîp

315 unt nâch gaten werken ringen, sô mac ime wol gelingen. swer aber in den sunden lit unde werltlicher êre phlit, daz her daz vleisch mestet

320 unde edel gwant an sich bestet: swer nâch des vleisches willen lebet unde nicht nâch gotes hulden strebet, der mûz zur helle, des dunket mich, des mac her nicht entsagen sich:

325 dâ mâz her inne beide brâten unde brinne, in deme hellischen vâre: da wirt ime alliz daz zu sûre, daz her ie begangen hât.

330 dâ hât ûch vor, daz ist min rât, unde vor allen dingen, die âch zur helle kunnen bringen, unde dînet umbe daz êwige leben. ich wil der rede ein ende geben.

Gotes mûter, der sundêr trôst, hilf uns, daz wir werden erlôst

310. er beschowe $B$. engele $A$. 311. Wir $A$, Ern $B$. habe ez niht e. $B$. ez fehlt $A$. 312. Da bi sei ein ieglich g. $B$. 313. weip oder man $B$. 314. Si svllen nach gvten werken stan $B$. 315. Dar n. svlle wir r. $B$. 316. So wirt vns $B$. 317. Wer $A$. 318. êre] vrevden $B$. 319. Swer daz $B$. 320. gewant $A$. Vñ riche kleider $B$. 321. Wer aber $A$. lebt : strebt $B$. 322. nach dem tode $B$. 323. zu d' helle $A B$. des fehlt $B$. 325. er immer innen $B$. 326. beide fehlt $B$. brinnen $B$. 327. dem $B$. fiwre $B$. 328. im $B$. savre $B$. 329. 30 . Des er hie was gewon hie hvte sich ein ielich von $B$. 330. hutet $A$. deaz $A$. 331 . Vnde hvte sich von a. $B$. allen den d. $A$. 332. uch] in $B$. zu der helle $A B$. mvgen $B$. 333. Unde fehlt $B$. 334. Hie wil ich $\operatorname{der} B$. 335. sundere $A$. 
von sunden unt von schande, daz wir heim zu lande komen alle vrôlîche

340 in daz schône himelriche. daz uns daz mûze geschên, sô sprechit alle âmên.

335-542. Des laz vns got mit selden leben vnde gebe vns dort daz immer lebē $B$. Dit is uiz ich enhain is neit me beschreven got brenge vns allen in dat ewige leven dat wir moizen aldae werden bekant mit allen heilgen in dat hemelsche lant des moiz uns gunnen der heilge geist und der vader mit dem sone allermeist ind dat dit geschei alzosamen zo ewigen ziden so sprecht alle amen $C$.

Aus der sammlung 'Geistliche gedichte des XIV. und XV. jahrh. vom niederrhein', welche Schade nach drucken aus dem beginnenden 16. jahrh. herausgegeben hat, sind zwei stücke ${ }^{1}$ ) schon von Luibben in älteren hss. aufgefunden und zum abdruck gebracht worden. Ein drittes, Der krantz der gotlicher lieffden Schade a. a. 0. s. 229-35, ist ausser in diessem Kölner drucke noch in zwei handschriften erhalten, mit deren zuhulfenahme in vorstehender ausgabe eine kritische herstellung des textes versucht werden konnte. Es bezeichnet

A die Leipziger pergamenths. des Sachsenspiegels (no. 946) in folio aus der zweiten hälfte ${ }^{2}$ ) des 14. jahrh., in

1) Van dem begyngyn van parisz Schade a. a. 0. 8. 337-56 und bei Lubben, Mittelniederdeutsche gedichte (Oldenburg 1868) no. I, s. 1-17 nach einer Oldenburger papierhs. des 15. jahrh. Zwoitens Sent Anselmus vrage tzo marien Schade a. a. o. s. 248-86 und in Lübbens Zeno, oder die legende von den heiligen drei königen. Ancelmus, vom leiden Christi (Bremen 1876) s. 103-44 nach einer Oldenburger papierhs. des 14. jahrh.

2) Ueber das alter der hs. bemerkt v. d. Hagen im Grundriss s. 399, dass sie 'gewis noch aus dem 14. jahrh.' stamme. Ebenso hat sie Moriz Haupt, der sich zwar einer abschrift Hoffmanns bediente (vgl. die folgende anm.), ohne zweifel aber auch die hs. selbst gekannt haben wird, ohne genauere angabe des früher oder später in dieses jahrhundert verwiesen, Altd. blätter I, s. 104. R. Hildebrand dagegen vindiziert sie schon in der dritten auflage des Sachsenspiegels s. XI anm. 'den schriftziggen und der ganzen haltung nach' mit bestimmtheit dem anfang des 14. jahrh. (und ihm folgend Weinhold, Mhd. grammatik 
welcher das gedicht auf bl. 60 vw. sp. a bis bl. 62 vw. sp. b gelesen wird. Eine umfängliche beschreibung derselben hat schon von der Hagen, im Grundriss s. 399-406, nebst angabe des einganges und schlusses der angehängten gedichte ${ }^{1)}$ gegeben, die indessen von Schade ubersehen worden ist. Aber auch von den auf s. 227 von letzterem zum beweise für die niederrheinische herkunft des gedichtes aus dem druck angezogenen reimen würde nur der eine ersein (ersehen) : ein v. 123. 24 geltung haben, wenn er nicht einem niederrheinischen abschreiber zur last gelegt werden muste, vgl. oben v. 219. 20; denn leit $\mathbf{v} .26$ ist jacet, nicht patiebatur, dinc : kint $\mathbf{v}$. 41.42 ist nicht specifisch niederrheinisch und gevêre (hêre $\mathrm{AB}$ ) : sêre v. 231. 32 ein guter mitteldeutscher reim. Eine sorgfältige und genaue copie dieser handschrift danke ich herrn gymnasiallehrer T. Hayner;

$B$ die Heidelberger pergamenths. no. 341 in folio, ebenfalls dem 14. jahrh. angehörig, bl. 78c-bl. $80 \mathrm{~d}$;

C den Kölner druck vom jahre 1513. Vgl. Schade a. a. 0 . s. $225 \mathrm{f}$.

Die mundarten, in denen diese drei texte niedergeschrieben wurden, sind nach der heimat ihrer schreiber verschieden.

s. 482) und bemerkt zur vierten auflage ebenda, dass eine vergleichung der sprachformen mit denen in den urkunden der stadt und des stiftes Meissen deutlich noch für das 13. spräche. Allein wenn schon die Leipziger Sachsenspiegelhs. no. 946 noch eine recht hübsche genannt werden darf, so ist sie doch in bezug auf feines pergament, eleganz und sauberkeit der schrift und künstlerische ausstattung keineswegs so prächtig, dass ihr nicht eine ziemliche anzahl zum teil schひnerer aus dem 14. jahrh. an die seite gestellt werden künnte. Auch zeigon ihre schriftzlige schon nicht mehr die scharfe fraktur, welche das besondere kennzeichen der hss. des 13. jahrh. ist, sondern die stumpferen und breiteren züge des 14. Den sichersten terminus a quo der entstehung dieser hs. ergibt aber die schon von $\mathrm{Homeyer}$ gemachte beobachtung, dass sie zur Buchschen recension gehørt, der zufolge sie denn auch von diesem den dieser gattung angehörenden sämmtlich in die zweite hälfte des 14. jahrh. fallenden texten beigesellt worden ist.

1) Von den fünf gedichten der hs. sind meines wissens bisher nur zwei veröffentlicht, das füfte Der spiler durch Hoffmann v. F. in den Altd. blättern I, s. 63-65 und das erste Spiegel der tugende durch Haupt mit herbeiziehung der Dresdener papierhs. $M, 68$ in folio vom jahre 1447, ebenda I, s. 88-104. 
$\mathrm{AB}$ geben nämlich die mitteldeutsche, jedoch $\mathrm{A}$ das nördliche nächst der niederdeutschen grenze gesprochene idiom, B ein mehr studliches: $C$ ist dagegen aus der feder eines niederrheinlănders geflossen. Die reime beweisen, dass auch der verfasser des gedichtes ein mitteldeutscher war und dass die sprache der hs. A der seinigen sehr nahe steht. Man vergleiche vân: hân 3. 4; gestalt : salt 79. 80; blêch :wêch 93. 94; breche : besteche 155. 56 ; niet : siet 181. 82; bie : sie 187 . 88; brechen : bestechen 217. 18; vor : tor 229. 30 und 239. 40; nicht : licht 277. 78; vure: sûre 327. 28. Eine eigentümlichkeit des Mitteldeutschen sind auch die apokopierten infinitive beginne: gewinne 5. 6; (clage:) sage 19. 20; (danne:) zanne 56. 57; lâze (:strâze) 137. 38; nenne : erkenne 163.64; (Mâze:) lâze 193. 94 ; weine (: eine) 221. 22 und (inne :) brinne 325. 26. Unter den reimen sind ausserdem noch folgende assonanzen zu beachten: erstens vocalische gebâren : gevaren 75. 76 (vorausgesetzt, dass diese beiden nur in A erhaltenen verse echt sind); glich : dich 97. 98; himelrich : sich 153. 54 und 253. 54; zweitens consonantische: Cristum : sun 105. 6; dinc: kint 41. 42; : sint 131. 32; twanc : lant 255. 56.

Die unabhängigkeit der drei handschriften unter einander ergibt sich schon aus dem bestande ihrer texte. Es fehlen nämlich in A v. $131-36,149-52,259-62$; in $B$ v. 5 . 6 $109-112,119,125.26$; in $\mathrm{C}$ v. $1-4,31-34,49.50$; 53-108, 145, 147, 167-72, 189-92, 199. 200, 207. 8, 221. 22, 251. 52, 255. 56, 271-334. Zwischen BC bestehen jedoch engere verwantschaftliche beziehungen, das beweist die grosse anzahl ihnen gemeinsamer und zum teil fehlerhafter lesarten, von denen ich die wichtigsten anfuhre. V. 18 sunden (sunde A); 42 ihesum christum B, Jesus Christus C (Christ. fehlt A); 113 mit (bi B) der warheit (der fehlt A). In allen drei fällen wird die lesart von A durch den vers gefordert. Bedeutender ist das fehlen des verbums (sint A) v. 209. 10; v. 166, wo B daz ist ein blvme daz si evch geseit und $\mathrm{C}$ dese blomen sint angeseit lesen, aber A daz ist ein blume sô gemeit gewis die echte lesart bewahrt. V. 140-42, wo BC lesen

Edele blvmen (menich edel bloemgin $C$ ) wol getan, die im den weck (eme einen sachten wech $C$ ) bespreiten vnd senften wek (einen soezen gank $C$ ) bereiten, 
dagegen A

$$
\begin{aligned}
& \text { qwelf blumen wol getan, } \\
& \text { die ime den weg gebreiten } \\
& \text { unde in wol geleiten, }
\end{aligned}
$$

und man sich unbedenklich fur die tuberlieferung in A entscheiden wird, obschon gebreiten in dieser bedeutung nicht gerade sehr häufig ist. V. 220 lautet in B der wil ich vil nahen des besten iehen,

mit stärkerer änderung in C

und in $\mathrm{A}$

gerne weinen van den besten ein

der mûz ich alles gûtes jên,

wodurch der pleonasmus mit v. 222 vermieden ist. Vgl. auch v. 228, wo sich derselbe ausdruck in B widerholt. Ferner v. 153-55, die in A folgende fassung haben

swer ûf den wec zu himelrich

kumen wil, der vlize sich, daz her dî blûmen breche,

\section{in $B$}

Dar vmbe so vleize sich in $\mathrm{C}$

soe vlize sich dairzo ein iglich, der dair begert den wech zom

hemelrich u. s. w.

und endlich v. 249. 50, wo der wortlaut in A ouch gûten smac unde edelen ruch hât die blûme âne allen bruch

widerum zweifach umgewandelt wurde, nämlich

\section{in $B$}

Si gibet vns vil svzen smak

vnd svzen rvch als si wol mack, in $\mathrm{C}$

und gift uns up erden ouch soeze smach und guide rouch.

In beiden stellen ist die thbereinstimmung in $\mathrm{BC}$ in dio augen fallend und besonders bei der zweiten die lesart von $A$ unverkennbar die ursprüngliche. Was zu den änderungen in $\mathrm{BC}$ resp. deren vorlage veranlassung gegeben, ist nicht recht $\mathrm{zu}$ ersehen; bei v. 249. 50 war es vielleicht der ausdruck âne allen bruch, welcher dem abschreiber anstoss erregte, da bruch in beiden handschriften beseitigt ist.

Allerdings finden auch zwischen AC mehrmals bemerkenswerte ubereinstimmungen statt. So v. 22 von (syn $\mathrm{C}$ ) sunden (fehlt B); 40 in den sunden (den fehlt B); 112 iemerlichen A, jamerlicher C (v. 111. 12 fehlen B); 175 gehorsamekeit A, 
gehoirsamheit C (gehorsam B); dieselben sind jedoch nicht der art, dass eine specielle verwantschaft von AC aus ihnen gefolgert werden dürfte.

Obschon A der zeit und dem orte der entstehung des gedichtes ohne zweifel sehr nahe steht, ist es doch nicht wahrscheinlich, dass sie unmittelbar aus dem original entstanden ist. Von geringfligigeren, $\mathrm{ABC}$ gemeinsamen fehlern absehend, hebe ich nur die tiefere verderbnis in v. 165 hervor, wo weder A ein kuscher munt, noch $\mathrm{C}$ kuische liefde das richtige haben und die lesart von B Eine heizet lop völlig nichtssagend ist. Gemeint ist offenbar 'keuschheit des leibes' und auf lip weist sowol lop $\mathrm{B}$, als liefde $\mathrm{C}$, so dass sich ein kûscher lip mit sicherheit als das echte erkennen lässt.

Demnach ist das handschriftenverhältnis so zu construieren:

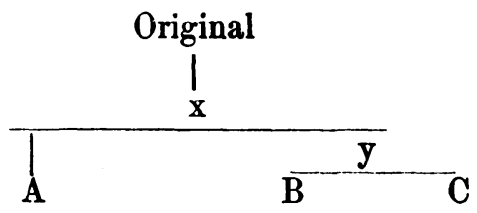

Zur charakteristik der einzelnen hss. bemerke ich noch folgendes. $\mathrm{C}$ ist nicht nur sehr lickenhaft, sondern auch durchweg so stark uberarbeitet, dass die vergleichung einer beliebigen stelle mit $\mathrm{AB}$ genulgt, $\mathrm{um}$ sich von dem geringen kritischen werte dieser hs. zu uberzeugen. Ebenso ist auch B von willkurlichen und den originalen text wesentlich umgestaltenden anderungen nicht frei. Ersichtlich ist dies schon in $\mathbf{v}$. 4, wo fur gotis hulde aus v. 6 daz himelrich eingesetzt wurde, um dann v. 5. 6 als uberflussig auszulassen. Ganz eclatant ist aber die beseitigung von reimen, welche der mundart des schreibers nicht gemäss waren und die er, um sie dieser anzubequemen, entweder durch andere reimwörter ersetzte, wie in v. 63. 64 trân: gehân A, trehen: sehen B und 185. 86 stêt : barmherzekêt AC, gemeit : barmherzikeit $\mathrm{B}$, oder durch einfügung ganz neuer verse aus dem wege räumte, wie mit auslassung von v. 61 nach 62 , um sundêr: richtêr und nach 155 und 156, um breche : besteche zu entfernen, während er den letzteren reim in v. 217. 18 unbeanstandet gelassen hat. Andere gründe, die vielleicht bloss in seinem besonderen geschmack 
zu suchen sind, bewogen ihn bei v. 35. 36, 54, 101-105, 119-22, 129. 30, 145-48, 171. 72, 203. 4, 271. 72, 286, 291. 92, 313-15, 329. 30 und 335-42 von seiner vorlage abzugehen. Die umstellungen von 205 und 206,299 und 300 sind dagegen wol als blosse versehen zu betrachten. Hiernach macht es keine schwierigkeit, die allein in $B$ vorkommenden verse, deren zwei nach 68, je vier nach 130 und 148, zwei (=199. 200) nach 180 und 26 nach 136 sich finden, als selbständige zusätze desselben schreibers zu nehmen. Die hinter v. 148 eingeschobenen vier verse sind ersichtlich in folge der unmittelbar voraufgehenden umdichtung von v. 145-48 entstanden, und die grössere interpolation nach v. 136 hat hauptsächlich später auftretende dinge vorweg genommen.

In $A$ lassen sich dagegen eigene veränderungen von einiger bedeutung nicht nachweisen. Die mängel dieser hs. bestehen meist in fluchtigkeiten des abschreibers (vgl. Hildebrand, Sachsensp. s. XI f.) und konnten daher gewöhnlich durch einfache mittel gebessert werden.

Bei der reconstruierung des textes war mithin A zu grunde zu legen, BC aber erst in zweiter linie herbeizuziehen.

Zum schlusse will ich noch auf einige anklänge an ältere oder ungefähr gleichzeitige gedichte hinweisen, die indessen wol mehr als einzelne im gedächtnisse des verfassers haftende reminiscenzen aus seiner lectüre, denn als directe entlehnungen anzusehen sein mögen. V. 67 steht ebenso im Leben $d$. heil. Franciscus (vgl. Pfeiffer, Altd. ubungsb. s. 60 ff.) v. 59 daz si dir herre got geklaeit. Ferner ist zu vergleichen v. 15. 16 mit Francisc. 76-78 daz ich mich bekere mit riwe von der missetat die min lip begangen hat; v. 321. 22 findet sich schon bei Reinmar (Wackernagel leseb. $I^{5}$ s. 506) Vil maneger nâch der werlte strebet, dem sî doch boesez ende gebet, und nieman weiz, wie lange er lebet, und dann öfter, z. b. im Helmbrecht (Haupt in seiner zschr. IV, s. $321 \mathrm{ff}$.) 519-26 sun, vil schœener jüngelinc, du solt sagen mir ein dinc (ob dir wonent witze $b \hat{\imath}$ ), welher baz lebender sî, dem man fluochet unde schiltet und des al diu welt engiltet und mit der liute schaden lebet und wider gotes hulde strebet, und daselbst v. $973 \mathrm{ff}$. der ist $n \hat{u}$ der wîse, der lôsen unde liegen kan,... leider michels mêre danne 
ein man der rehte lebet und nâch gotes hulden strcbet; vgl. noch Warnung (Haupt in seiner zschr. I, s. 438 ff.) v. 2269. 70) und Fiancisc. 177. 78. Mit br. Philipps Marienl. v. 8678. 79 dâ Jêsus Christus vïert den tanz und treit von bluomen einen kranz sind zu vergleichen v. 157. 58 und mit br. Phil. จ. 8697-99 diu spîse ist ouch sô gestalt, swem sî kumt in sînen munt, der blibet immer mêr gesunt v. 201. 2 unseres gedichtes. WOLFENBÜTTEL. GUSTAV MILCHSACK. 